\title{
BMJ Open Young Survivors at KSA: registry for standardised assessment of long-term and late-onset health events in survivors of childhood and adolescent cancer $-\mathbf{a}$ study protocol
}

Maria Otth (D) , ${ }^{1,2}$ Daniel Drozdov (D) , ${ }^{1,2}$ Claudia Hügli, ${ }^{1}$ Katrin Scheinemann (i) ${ }^{1,3,4,5}$

To cite: Otth M, Drozdov D, Hügli C, et al. Young Survivors at KSA: registry for standardised assessment of long-term and late-onset health events in survivors of childhood and adolescent cancer-a study protocol. BMJ Open 2021;11:e053749. doi:10.1136/ bmjopen-2021-053749

- Prepublication history for this paper is available online. To view these files, please visit the journal online (http://dx.doi org/10.1136/bmjopen-2021053749).

$\mathrm{M} 0$ and $\mathrm{DD}$ contributed equally.

Received 22 May 2021

Accepted 11 November 2021

Check for updates

(C) Author(s) (or their employer(s)) 2021. Re-use permitted under CC BY-NC. No commercial re-use. See rights and permissions. Published by BMJ.

For numbered affiliations see end of article.

Correspondence to

Dr Daniel Drozdov;

daniel.drozdov@ksa.ch

\section{ABSTRACT}

Introduction A high proportion of survivors of childhood and adolescent cancer experience chronic medical conditions - late effects. Most studies on late effects have a retrospective or questionnaire-based design, which leads to unavoidable limitations such as missing data or different severity coding and grading of late effects. We, therefore, need prospective data, including standardised severity coding and grading. 'Young Survivors at KSA' aims to close this gap by assessing frequency, severity, risk factors and longitudinal changes of late effects in childhood cancer survivors prospectively and in a standardised way.

Methods and analysis Within the 'Young Survivors at KSA' registry, we collect data from regular follow-up visits in a comprehensive database prospectively and repeatedly from start of the study and retrospectively at most until January 2016. We classify and grade the severity of late effects according to the Common Terminology Criteria for Adverse Events version 4.0 modified by Hudson et al. The outcome variables correspond to results from risk-stratified organ examinations, performed according to the Children's Oncology Group guidelines version 5.0 and the recommendations by the International Guideline Harmonization Group. We collect the exposure variables from the patients' medical history, including detailed information on cancer diagnosis and treatment. We analyse the data in an exposure-driven and organ systemdriven approach. We start recruitment with patients treated at the Kantonsspital Aarau, Switzerland. However, our design allows the inclusion of additional national centres later.

Ethics and dissemination 'Young Survivors at KSA' is approved by the Ethikkommission Nordwest- und Zentralschweiz, reference number A0_2020-00012. The results of this study will be presented at scientific meetings, including meetings with childhood cancer survivors and published in peer-reviewed and if possible open access journals. New insights gained from the study will be used directly in clinical practice.

Trial registration number ClinicalTrials.gov NCT04811794; https://clinicaltrials.gov/ct2/show/study/ NCT04811794
Strengths and limitations of this study

- 'Young Survivors at KSA' is a prospective study with a registry design, additionally including retrospective data from 2016 to 2021.

- We collect baseline data, assess and code late effects in a standardised way directly from electronical medical records with full access to treatment data and documentation of follow-up visits.

- We include childhood cancer survivors initially from our institution only, where we established a well-organised transition process from paediatric to adult-focused follow-up care; later we plan to include patients from additional centres within Switzerland.

- We use a secure electronic web-based data capturing system facilitating data collection of repeated visits, including results from clinical examination, laboratory and function tests.

- We collect data obtained during routine clinical care and do not specify the examinations that need to be done, which might result in missing data.

\section{INTRODUCTION}

Childhood cancer survivorship

Around 350 children and adolescents up to the age of 20 years are diagnosed with cancer annually in Switzerland. ${ }^{1}$ Until 18 years of age, patients are treated in one of the nine certified paediatric oncology centres (Aarau, Basel, Bellinzona, Bern, Geneva, Lausanne, Lucerne, St. Gallen and Zurich), united in the Swiss Paediatric Oncology Group (SPOG). In Switzerland, the 10-year survival rate of children and adolescents diagnosed with cancer now exceeds $87 \%$ for all types of cancer combined. ${ }^{2}$ This results in increasing numbers of long-term childhood cancer survivors (CCSs). Currently, around 7000 CCSs live in Switzerland. However, many CCSs are diagnosed with chronic medical conditions, so called late effects, due to the cancer itself 
Table 1 Examples of organs commonly affected through specific treatment modalities, not exhaustive

\begin{tabular}{|c|c|}
\hline Affected organ & Treatment modality \\
\hline Heart & $\begin{array}{l}\text { Chemotherapy with anthracyclines, } \\
\text { radiation to the chest, abdomen, spine } \\
\text { (thoracic, whole), TBI }\end{array}$ \\
\hline Lung & $\begin{array}{l}\text { Chemotherapy with bleomycin, busulfan, } \\
\text { lomustin, carmustin, radiation to the chest } \\
\text { and axilla, TBI, HSCT with history of } \\
\text { chronic GvHD, thoracic surgery }\end{array}$ \\
\hline Ear & $\begin{array}{l}\text { Chemotherapy with carboplatin, cisplatin, } \\
\text { radiation to the head/brain, TBI }\end{array}$ \\
\hline Kidney & $\begin{array}{l}\text { Chemotherapy with ifosfamide, } \\
\text { carboplatin, cisplatin, cyclophosphamide, } \\
\text { radiation to the abdomen, TBI, } \\
\text { nephrectomy, HSCT }\end{array}$ \\
\hline $\begin{array}{l}\text { Female } \\
\text { reproductive } \\
\text { system }\end{array}$ & $\begin{array}{l}\text { Chemotherapy with alkylating agents } \\
\text { (busulfan, cyclophosphamide, ifosfamide), } \\
\text { ovarian radiation, TBI, cranial radiation, } \\
\text { HSCT, unilateral oophorectomy }\end{array}$ \\
\hline $\begin{array}{l}\text { Male } \\
\text { reproductive } \\
\text { system }\end{array}$ & $\begin{array}{l}\text { Chemotherapy with alkylating agents } \\
\text { (busulfan, cyclophosphamide, ifosfamide), } \\
\text { cisplatin, testicular radiation, TBI, cranial } \\
\text { radiation, HSCT, orchiectomy }\end{array}$ \\
\hline
\end{tabular}

GvHD, graft-versus-host disease; HSCT, haematopoietic stem cell transplantation; TBI, total body irradiation.

or its treatment. ${ }^{3}$ Treatment modalities known to cause late effects include chemotherapy, haematopoietic stem cell transplantation, radiotherapy or surgery. ${ }^{45}$ They can cause late effects due to their off-target effects, as they do not only attack tumour cells but also healthy tissue. As a consequence, every organ system, for example, the respiratory, cardiovascular, sensorineural and endocrine system, skin, central nervous system or immune system, can potentially be affected by late effects. ${ }^{6}$ Depending on the cancer treatment received and individual factors, including age at treatment, each CCS has a different risk profile for developing late effects (table 1). Risk-adapted and organ-specific follow-up care is, therefore, needed, especially as many late effects are subclinical for a long time (e.g. pulmonary function impairment, cardiovascular disease) and CCSs are often unaware of their condition until clinical symptoms develop or a focused clinical examination is performed. ${ }^{3}$

Results from a large US cohort published in 2006 support the importance of long-term follow-up care over $70 \%$ of CCSs suffered from any late effect 30 years after completion of treatment. ${ }^{7}$ In a more recent publication from 2017, the cumulative incidence of any chronic health condition at the age 50 years reached $99.9 \%$ for all conditions and $96.0 \%$ for severe or disabling conditions, according to Common Terminology Criteria for Adverse Events (CTCAE) grades $3-5 .{ }^{8}$ These data highlight that long-term follow-up care into adulthood is of outmost importance for CCSs. In this context, several survivor cohorts have already been established. The Swiss Childhood Cancer Survivors Study (SCCSS) ${ }^{9}$ the Childhood Cancer Survivor Study (CCSS) in the USA, ${ }^{10}{ }^{11}$ the British Childhood Cancer Survivor Study (BCCSS), ${ }^{12}$ the Nordic Adult Life after Childhood Cancer in Scandinavia(ALiCCS) ${ }^{13}$ and the French COHOrt of the PEdiatric cancer Registries (COHOPER) ${ }^{14}$ are either purely questionnaire-based or a combination of questionnairebased and linkage with routine data. These large cohorts and data sets are very rich in information, ${ }^{15}$ but they harbour some disadvantages when it comes to the objective quantification of medical conditions. Self-reported questionnaire-based data are prone for participation and reporting bias-especially when it comes to medical diagnoses, for example, pneumonia, cystitis or the severity of hearing or vision impairment. Four survivor cohorts additionally include clinical data collected prospectively. These cohorts are the St. Jude Lifetime Cohort (SJLIFE) in the USA ${ }^{16}{ }^{17}$ the Dutch Childhood Oncology Group Program on Late Effects after Childhood Cancer (DCOG LATER) ${ }^{18}$ the French Leucémies de l'Enfant et l'Adolescent (LEA) cohort including leukaemia survivors, ${ }^{19}$ and the French solid cancers counterpart, the French Childhood Cancer Survivor Study (FCCSS) ${ }^{20}$ The recruitment of CCSs in these cohorts starts the earliest 2 years after completion of treatment in the LEA cohort, 5 years after completion in the FCCSS and DCOG LATER ${ }^{15}$ and 10 years after completion in the SJLIFE cohort. With these approaches, the baseline at entering follow-up care is missing. With 'Young Survivors at KSA', we aim to close this knowledge gap by recruiting CCSs directly at the time point of entering follow-up care. We additionally collect information from the follow-up visits prospectively, in a standardised way, and cover children and adolescents treated with novel targeted agents (e.g. blinatumomab) and immunotherapy.

\section{Recommendations for long-term follow-up care}

Recommendations for follow-up care after completion of treatment are part of international treatment protocols. However, they are outlined in detail for a maximum of up to 10 years and only vaguely, thereafter, are not specified for different treatment arms within the protocols and focus on the detection of relapse more than of late effects. Based on the increasing knowledge on the development of late effects years to decades after completion of treatment, international guidelines for long-term follow-up care, such as the Children's Oncology Group (COG) guidelines from the USA, ${ }^{5}$ the Stichting Kinderoncologie Nederland guidelines from the Netherlands ${ }^{21}$ or the 'Therapy-Based Long-Term Follow-up Guidelines' from the UK, ${ }^{22}$ have been published and are steadily updated. Although the recommendations from the different national guidelines largely overlap, there exist nevertheless differences. The international effort within the International Late Effects of Childhood Cancer Guideline Harmonization Group (IGHG) aims to harmonise the follow-up care guidelines internationally, to optimise 
quality of care, to improve survivor's quality of life and to reduce duplication efforts. ${ }^{4}$ For the purpose of this registry, we rely on the COG Long-Term Follow-Up guidelines $^{5}$ and the IGHG recommendations ${ }^{4}$ to define the individual risk of each CCS and to formulate screening recommendations.

\section{Assessment of late effects}

Late effects detected during follow-up care visits are often described to each physician's best knowledge and 'standard of care', but they might differ between centres, between departments (paediatric vs adults) and over time. To be able to combine medical data on late effects over a longer period and from different centres, a standardised and uniform recording is necessary. The CTCAE is a widely used descriptive terminology designed to report adverse events of each organ system and it additionally provides a grading scale for each adverse event. ${ }^{23}$ However, the CTCAE criteria do not fully meet the needs of CCSs. The events assessed in the CTCAE are not completely representative of the spectrum of events experienced by CCSs, and the CTCAE criteria do not routinely integrate detailed patient-reported outcomes and medical outcome data available from clinically assessed cohorts. To overcome these shortcomings, Hudson et al from the SJLIFE addressed these deficits and published modified CTCAE criteria, which we use for the 'Young Survivor at KSA' registry (https://cebp.aacrjournals.org/content/ suppl/2016/12/29/1055-9965.EPI-16-0812.DC1). ${ }^{6}$

\section{METHODS AND ANALYSIS}

\section{Study design and population}

'Young Survivors at KSA' is a registry with a prospective design. The study population consists of CCSs, defined as former childhood cancer patients who were diagnosed before age 18 years, completed their cancer treatment at recruitment and entered follow-up care. At time of recruitment, these CCSs can still be children, adolescents or adults already. The recruitment starts in the Division of Oncology-Haematology, Department of Paediatrics, Kantonsspital Aarau. Here, we assess the transition readiness of CCSs starting at the age of 15 years on a regular basis. ${ }^{24}$ If the CCS feels ready, we implement a transition process to adult-focussed care, including joint consultations with members of paediatric and adult team over at least two annual visits. ${ }^{25}$ Every CCS receives a 'survivorship care plan' with detailed information about individual cancer treatment, risks for late effects and recommended health screening. ${ }^{26}$ Additional centres will be added later.

Depending on where the survivors are in their follow-up care at recruitment, we defined two distinct groups within the study population. CCSs in group A will make up the majority of the study population and represent CCSs who are still in follow-up care at the time of recruitment. CCSs in group B will represent a small part of the study population and include those who are not in follow-up care

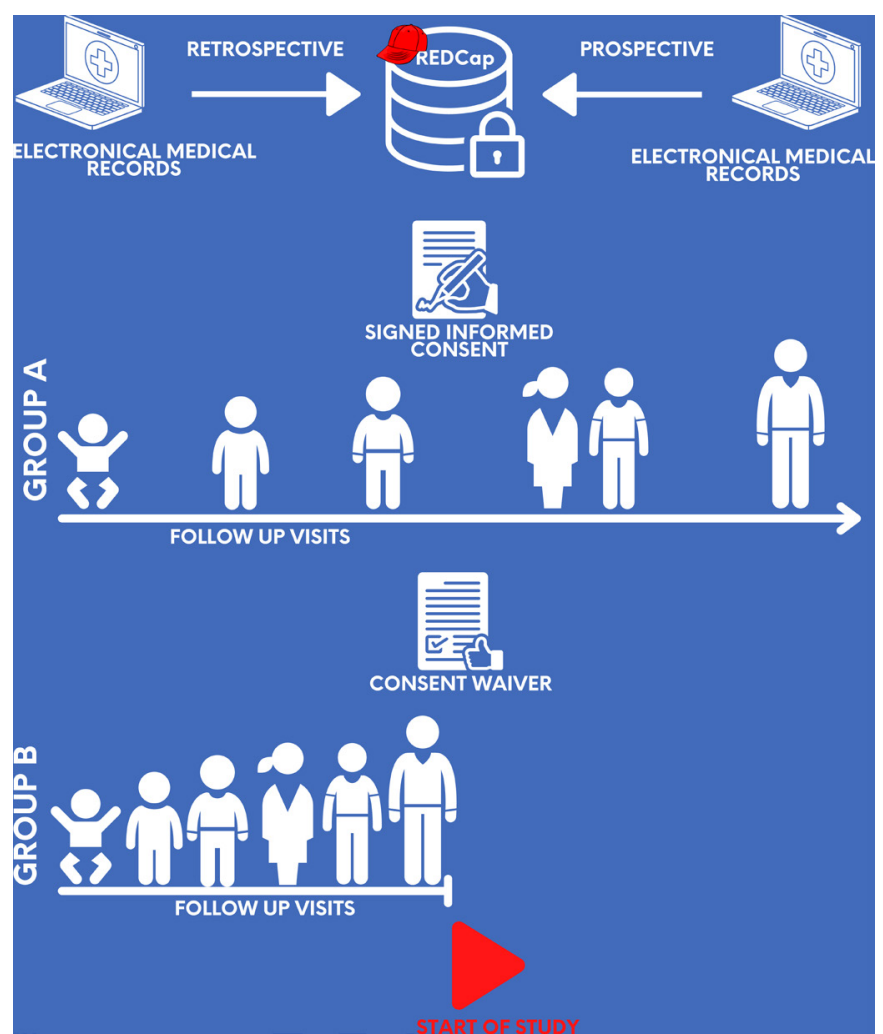

Figure 1 Registry outline with recruitment timeline, consent and follow up for childhood cancer survivors still in follow-up care (group A) and childhood cancer survivors who finished follow-up care (group B).

anymore, because they left follow-up care on purpose, moved away or died (figure 1).

\section{Patient and public involvement}

We did not involve patients and CCSs in setting up the registry, as the tests to be performed during follow-up care are given by the long-term follow-up care guidelines and the standardised coding by the modified CTCAE criteria. However, when it comes to defining research areas and disseminating results from the registry data, CCSs will be included.

\section{Inclusion and exclusion criteria}

Group A includes CCSs who were treated for their cancer in the Department of Paediatrics, Kantonsspital Aarau, later in a participating SPOG centre, have been diagnosed at age between 0 and 18 years, are still in regular follow-up care at Kantonsspital Aarau, later the participating SPOG centre, have finished their cancer treatment, entered follow-up care and signed the informed consent. Group B includes CCSs who fulfil the same inclusion criteria as group A but are not in regular follow-up care anymore. CCSs were excluded, if they were in palliative care, have not completed their cancer treatment or have not given informed consent for further use of their medical data.

\section{Sample size}

The number of survivors participating in 'Young Survivors at KSA' determines the sample size. We estimate around 
300 CCSs fulfilling the inclusion criteria at the Kantonsspital Aarau when opening the registry and another 25 CCSs who will annually newly qualify. As soon as other SPOG centres will join, the sample size will augment accordingly.

\section{Primary outcome measures}

The primary outcome corresponds to test results from organ-specific follow-up examinations assessed longitudinally, for example, echocardiography. If late effects are detected, their presence and severity are graded according to the modified CTCAE criteria. With the example of CCSs at risk for cardiac late effects, the modified CTCAE criteria cover congestive heart failure, heart valve disorder, left or right ventricular systolic dysfunction and their respective severity.

\section{Secondary outcome measures}

Secondary outcomes correspond to characteristics needed to describe the study population or risk factors that might have an impact on the development of late effects. These secondary outcomes include:

1. Sociodemographic and socioeconomic characteristics, such as age at clinical examination, gender, education or working situation, living situation.

2. Comorbidities, such as arterial hypertension or obesity.

3. Information related to the diagnosis, such as age at diagnosis, diagnosis, disease stage or treatment protocol.

4. Information related to the treatment, such as cumulative doses of chemotherapeutic agents and radiotherapy, surgery or haematopoietic stem cell transplantation.

\section{Data collection}

Data collection and data entry in the registry-specific database will be performed by dedicated and trained study members from Kantonsspital Aarau, including paediatric oncologists and study nurses. The data will directly be entered in the database without primarily completing a case report form. Data will be collected for each CCS in annual intervals, since all CCSs normally receive an annual comprehensive examination. This applies to CCSs who just entered follow-up care, where data from the most comprehensive examination will be used as follow-up visits are more frequent than annually, and to CCSs who are in follow-up care for several years already.

Of CCSs from group A and group B data on personal history, diagnosis, treatment, socioeconomic factors, physical examinations, diagnostic tests and laboratory tests will be collected as per clinical practice. Detailed information and examples of data collected within this registry are described in box 1 .

For CCSs in group A, we will collect data retrospectively till 2016 and prospectively starting from the recruitment in the registry. No end is foreseen to stop the data collection. We chose the year 2016 as the furthest back since this year onward, data from all patient visits are recorded electronically. In addition, this is 5 years before starting the registry and the further back the data are collected,
Box 1 Overview over parameters that can be recorded in the registry-specific database

\section{Personal data.}

Month and year of birth.

Sex.

Personal and family history relevant for late effects.

Known hearing impairment before cancer diagnosis.

Cancer predisposition syndromes.

Month and year of death (if applicable).

Data related to cancer diagnosis and treatment.

ICD10 diagnosis.

ICCC3.

Date of diagnosis.

Date of relapse (if applicable).

Tumour localisation.

Name of treatment protocol, including treatment arm (if applicable).

Information on classical chemotherapeutic agents, novel targeted agents and immunotherapy (if applicable).

Name of agent.

Cumulative dose.

Information on radiotherapy (if applicable).

Involved region and cumulative dose.

Information on surgery (if applicable).

Information on haematopoietic stem cell transplantation and CAR-T cell treatment (if applicable).

Relevant current medications (e.g. hormone replacement therapy after cranial radiotherapy).

\section{Data related to follow-up visits.}

Date of follow-up visit.

Results from organ-specific physical examination, e.g. blood pressure, weight and height.

Results from functional tests, e.g. hearing threshold, pulmonary function.

Bone densitometry (DEXA).

Results from laboratory tests.

Blood (e.g. renal function, hormonal levels).

Urine (e.g. proteinuria).

Cerebrospinal fluid (if applicable).

Results from pathology reports.

Results from imaging.

Ultrasound.

Magnetic resonance imaging (MRI).

Computed tomography (CT).

Current medications to treat late effects

Abbreviations:

CAR-T cells, chimeric antigen receptor T-cells; ICD10, International Classification of Disease Version10; ICCC3, International Classification of Childhood Cancer Version 3.

the higher becomes the risk for bias. The data sources are, therefore, primarily the electronic patient records. We will search the paper archives only for the retrospective part if data cannot be found in the electronic records. For CCSs in group B, we will collect data only retrospectively, between 2016 and the last contact with the respective survivor. We expect the impact of CCSs from group $\mathrm{B}$ with individual missing outcome parameters to be very small, as the group per se will be much smaller than 
group A and will make up an ever-smaller proportion as the total cohort grows. The participant timelines of CCSs from group A and group B are depicted in figure 1 .

\section{Data management}

Study members enter the collected data in the dedicated, secure and web-based data capturing system REDCap (Research Electronic Data Capture). ${ }^{27} 28$ The REDCap database for 'Young Survivors at KSA' is hosted at the Clinical Trial Unit of the University of Bern. REDCap provides audit trails to track changes in entered data and the export procedures. The REDCap database of 'Young Survivors at KSA' consists of 19 different modules-one module to verify CCSs' eligibility, one module to enter information on diagnosis and treatment very comprehensively, one module for general information collected during every follow-up visits, such as height, weight or current medication use, one end of study form, which has to be completed in case a participant leaves follow-up care, and one module dedicated for each organ system or late effect listed in the modified CTCAE criteria (https://cebp.aacrjournals.org/content/suppl/2016/ 12/29/1055-9965.EPI-16-0812.DC1). ${ }^{6}$ The included organ systems or late effects are the following: neuropsychological and psychological health, auditory/hearing, cardiovascular, endocrine, gastrointestinal, hepatobiliary, haematological, immunological, infectious, musculoskeletal, neurological, ocular/visual, pulmonary, renal/ urinary tract and reproductive/genital health. ${ }^{29}{ }^{30}$ Study members from Kantonsspital Aarau have access to the database. Each member has an individual account. When additional SPOG centres will participate in the future, separate and secure access will be provided to these study members and members of each centre will only have access to their own survivors.

\section{Statistical methods}

We will apply descriptive statistics, such as mean and standard deviation, median and range, summary tables and graphics, to describe the study population and the prevalence and severity of late effects in CCSs according to the modified CTCAE criteria. To examine the differences between groups, for example, between CCSs with different severities of the same late effect or CCSs exposed and not exposed to risk factors, we will apply statistical tests depending on the type of variable in question. These tests may include t-test or Mann-Whitney test for continuous outcome variables or $\chi^{2}$ or Fisher's exact test for categorical outcome variables. For more advanced analyses of associations between the outcomes and covariates of interest, we will use appropriate regression models adjusted for possible confounders, including age, sex, height or type of cancer. The data set will give us the possibility to collect time-to-event data, which will be analysed non-parametrically using the Kaplan-Meier method. Associations with covariates will be modelled using Cox regression. For repeated data, for example, results from repeated echocardiograms, we will apply statistical methods for longitudinal data, such as mixed effects models. As software, we will use Stata Statistical Software (STATA Corp LLC, Texas, USA) and R (R Foundation for Statistical Computing, Vienna, Austria).

\section{Ethics and dissemination}

The 'Young Survivors at KSA' was approved by the cantonal ethics committee EKNZ (Ethikkommission Nordwest- und Zentralschweiz; AO_2020-00012) and is registered on ClinicalTrials.gov.

Written informed consent to participate is obtained from all participants from group A. Children below the age of 14 years receive an age-appropriate information describing the registry, the consent is signed by the parents. Adolescents aged 14-18 years and adults, 18 years of age and older, sign the informed consent themselves. The study information and informed consent is handed out during the next regular follow-up visit of eligible CCSs or is sent home. By signing the informed consent, participants agree that their data collected in the 'Young Survivors at KSA' registry can be further used for research, analysis and publications. Consent waiver was given from the cantonal ethic committee for participants from group B, according to Swiss Federal Act of 30 September 2011 on Research involving Human Beings (Human Research Act). For these CCSs, the effort to obtain informed consent would be disproportionate, or even impossible, such as in case of moving abroad or deceased CCSs.

\section{Author affiliations}

${ }^{1}$ Division of Oncology-Haematology, Department of Paediatrics, Kantonsspital Aarau AG, Aarau, Switzerland

'Department of Oncology, Haematology, Immunology, Stem Cell Transplantation and Somatic Gene Therapy, University Children's Hospital Zürich, Zurich, ZH, Switzerland ${ }^{3}$ Department of Paediatrics, McMaster Children's Hospital, Hamilton, Ontario, Canada

${ }^{4}$ University of Basel, Basel, Switzerland

${ }^{5}$ McMaster University, Hamilton, Ontario, Canada

Contributors MO and KS conceived the study, led the proposal and protocol development. KS is the primary investigator of the study. DD, MO and $\mathrm{CH}$ wrote the manuscript. KS reviewed the manuscript. All authors read and approved the final manuscript.

Funding This work is supported by the research council (Forschungsrat) of Kantonsspital Aarau, Switzerland a public hospital owned by the Canton of Aargau, grant number 1410.000.113, after an independent peer-review by two experts.

Competing interests None declared.

Patient and public involvement Patients and/or the public were involved in the design, or conduct, or reporting, or dissemination plans of this research. Refer to the Methods section for further details.

Patient consent for publication Not applicable.

Provenance and peer review Not commissioned; externally peer reviewed.

Open access This is an open access article distributed in accordance with the Creative Commons Attribution Non Commercial (CC BY-NC 4.0) license, which permits others to distribute, remix, adapt, build upon this work non-commercially, and license their derivative works on different terms, provided the original work is properly cited, appropriate credit is given, any changes made indicated, and the use is non-commercial. See: http://creativecommons.org/licenses/by-nc/4.0/.

ORCID iDs

Maria Otth http://orcid.org/0000-0002-2839-502X 
Daniel Drozdov http://orcid.org/0000-0002-3010-7525

Katrin Scheinemann http://orcid.org/0000-0002-3578-7152

\section{REFERENCES}

1 Belle F, Pfeiffer V, Redmond S. Swiss childhood cancer registry annual report 2017 /2018. 41. Switzerland: Swiss Childhood Cancer Registry, 2018.

2 Schindler M, Belle FN, Grotzer MA, et al. Childhood cancer survival in Switzerland (1976-2013): Time-trends and predictors. Int J Cancer 2017;140:62-74.

3 Hudson MM, Ness KK, Gurney JG, et al. Clinical ascertainment of health outcomes among adults treated for childhood cancer. JAMA 2013;309:2371.

4 Guidelines. International guideline harmonization group. Available: https://www.ighg.org/guidelines/ [Accessed 26 Mar 2021]

5 Children's Oncology Group. Available: http://www.survivorshipguid elines.org/ [Accessed 16 Feb 2021]

6 Hudson MM, Ehrhardt MJ, Bhakta N, et al. Approach for classification and severity grading of long-term and late-onset health events among childhood cancer survivors in the St. Jude lifetime cohort. Cancer Epidemiol Biomarkers Prev 2017;26:666-74.

7 Oeffinger KC, Mertens AC, Sklar CA, et al. Chronic health conditions in adult survivors of childhood cancer. N Engl J Med 2006;355:1572-82.

8 Bhakta N, Liu Q, Ness KK, et al. The cumulative burden of surviving childhood cancer: an initial report from the ST Jude lifetime cohort study (SJLIFE). Lancet 2017;390:2569-82.

9 Kuehni CE, Rueegg CS, Michel G, et al. Cohort profile: the Swiss childhood cancer Survivor study. Int J Epidemiol 2012;41:1553-64.

10 Robison LL, Mertens AC, Boice JD, et al. Study design and cohort characteristics of the childhood cancer Survivor study: a multi-institutional collaborative project. Med Pediatr Oncol 2002;38:229-39.

11 Robison LL, Armstrong GT, Boice JD, et al. The childhood cancer Survivor study: a national cancer Institute-supported resource for outcome and intervention research. J Clin Oncol 2009;27:2308-18.

12 Hawkins MM, Lancashire ER, Winter DL, et al. The British childhood cancer Survivor study: objectives, methods, population structure, response rates and initial descriptive information. Pediatr Blood Cancer 2008;50:1018-25.

13 Asdahl PH, Winther JF, Bonnesen TG, et al. The adult life after childhood cancer in Scandinavia (ALiCCS) study: design and characteristics. Pediatr Blood Cancer 2015;62:2204-10.

14 COHOPER - RNCE. COHOPER - national cohort of the paediatric cancer registries. Available: https://rnce.inserm.fr/index.php/en/ plateform/national-cohort-of-the-paediatric-cancer-registries [Accessed 4 May 2021].

15 Winther JF, Kenborg L, Byrne J, et al. Childhood cancer Survivor cohorts in Europe. Acta Oncol 2015;54:655-68.
16 Howell CR, Bjornard KL, Ness KK, et al. Cohort profile: the St. Jude lifetime cohort study (SJLIFE) for paediatric cancer survivors. Int J Epidemiol 2021;50:39-49.

17 Hudson MM, Ness KK, Nolan VG, et al. Prospective medical assessment of adults surviving childhood cancer: study design, cohort characteristics, and feasibility of the St. Jude lifetime cohort study. Pediatr Blood Cancer 2011;56:825-36.

18 Skion Later Studie - SKION LATER Study. Available: https://www. skionlaterstudie.nl/english/43:skion-later-study/ [Accessed 4 May 2021].

19 Berbis J, Michel G, Baruchel A, et al. Cohort profile: the French childhood cancer Survivor study for leukaemia (LEA cohort). Int J Epidemiol 2015;44:49-57.

20 FCCSS | Le site de l'étude FCCSS. Available: https://fccss.fr/?lang= en [Accessed 4 May 2021].

21 SKION (Stichting Kinderoncologie Nederland). Richtlijn follow-up na kinderkanker: meer dan 5 jaar na diagnose : Deel 1. SKION, 2010.

22 Skinner R, Wallace WHB, Levitt GA. Therapy based long term follow up. 62. 2nd edn. Leister, UK: United Kingdom Children's Cancer Study Group Late Effects Group, 2005. ISBN: 0-7017-0185-4.

23 Common terminology criteria for adverse events (CTCAE) | protocol development | CTEP. Available: https://ctep.cancer.gov/ protocolDevelopment/electronic_applications/ctc.htm\#ctc_60 [Accessed 26 Mar 2021].

24 Otth M, Wechsler P, Denzler S, et al. Determining transition readiness in Swiss childhood cancer survivors - a feasibility study. BMC Cancer 2021;21:84.

25 Denzler S, Otth M, Scheinemann K. Aftercare of childhood cancer survivors in Switzerland: protocol for a prospective multicenter observational study. JMIR Res Protoc 2020;9:e18898.

26 Oeffinger KC, Hudson MM, Mertens AC, et al. Increasing rates of breast cancer and cardiac surveillance among high-risk survivors of childhood Hodgkin lymphoma following a Mailed, one-page survivorship care plan. Pediatr Blood Cancer 2011;56:818-24.

27 Harris PA, Taylor R, Thielke R, et al. Research electronic data capture (REDCap)--a metadata-driven methodology and workflow process for providing translational research informatics support. J Biomed Inform 2009;42:377-81.

28 Harris PA, Taylor R, Minor BL, et al. The REDCap Consortium: building an international community of software platform partners. $J$ Biomed Inform 2019;95:103208.

29 Mulder RL, Font-Gonzalez A, Hudson MM, et al. Fertility preservation for female patients with childhood, adolescent, and young adult cancer: recommendations from the PanCareLIFE Consortium and the International late effects of childhood cancer guideline harmonization group. Lancet Oncol 2021;22:e45-56.

30 Mulder RL, Font-Gonzalez A, Green DM, et al. Fertility preservation for male patients with childhood, adolescent, and young adult cancer: recommendations from the PanCareLIFE Consortium and the International late effects of childhood cancer guideline harmonization group. Lancet Oncol 2021;22:e57-67. 